\title{
Outcrossing rates and allozyme variation in rayed and rayless morphs of Bidens pilosa
}

\author{
M. Sun* and \\ Fred R. Ganders $\dagger$
}

\author{
* Department of Agronomy and Range Science, \\ University of California, Davis, CA, 95616, U.S.A. \\ + Department of Botany, University of British \\ Columbia, Vancouver, B.C., Canada V6T 2 B1.
}

Thirty-four isozyme loci were assayed in 1048 plants from three Hawaiian populations of the autogamous introduced weed Bidens pilosa. Total isozyme gene diversity was very low, $0 \cdot 049$. One population containing only rayless plants was completely monomorphic at all loci except PGI-3, and this locus was nearly fixed. In a large population polymorphic for rayed, rayless, and intermediate plants, two loci showed rare variants and PGI-3 was polymorphic. Allele frequencies at PGI-3 were not significantly different among the floral morphs, but outcrossing rates, measured by progeny tests using $P G I-3$ as a genetic marker, were significantly higher in the radiate morph ( 9 per cent) than in the rayless and intermediate morphs ( 5 per cent). Presumably radiate plants have higher outcrossing rates because they are more attractive to pollinators.

\section{INTRODUCTION}

Bidens pilosa L. (Asteraceae) is a cosmopolitan subtropical and tropical weed native originally to North, Central, and South America (Ballard, 1986). Usually the flowering heads are discoid (without ray florets) and relatively inconspicuous, only 6-7 mm wide. However, a morph with 4-7 white ray florets with ligules $5-7 \mathrm{~mm}$ long is also common throughout most of the range of the species. The flowering heads of this morph are $12-15 \mathrm{~mm}$ wide, which is still relatively small for species of Bidens, but they are considerably more conspicuous than the rayless morph. The radiate form is sometimes distinguished taxonomically as var. minor, but appears to differ in no other ways from the rayless form, and both frequently occur intermixed in the same population.

The major function of ray florets in most members of the Asteraceae is usually assumed to be pollinator attraction, and many rayless species are thought to be predominantly self pollinating. Consequently, one would predict that a radiate morph would have a higher outcrossing rate than a rayless morph. However, there is little quantitative data available to test whether the presence of ray florets alone can affect pollinator visits or outcrossing rates. Populations polymorphic for radiate and rayless plants should provide a better experimental system for testing the effects of ray florets on outcrossing rates than comparisons between species, because other features of the mating system are less likely to vary between morphs than between species.

Several studies of populations of Senecio vulgaris L. polymorphic for radiate and rayless plants have shown that the radiate form has a significantly higher intermorph outcrossing rate than the rayless form (Marshall and Abbott, 1982, 1984a). This, in part, has been attributed to the effect of the ray florets in pollinator attraction (Abbott and Irwin, 1988). However, there are several complicating factors in this polymorphism in Senecio. The radiate morph was derived by introgressvie hybridisation from the radiate species $S$. squalidus L. within very recent times. The ray florets of $S$. vulgaris are pistillate and cannot automatically self pollinate (Marshall and Abbott, 1984b).

It therefore seems desirable to test the hypothesis that radiate morphs have higher outcrossing rates than rayless morphs in other species in the Asteraceae. We have estimated outcrossing rates and genetic variability at isozyme loci in populations of $B$. pilosa, to test this hypothesis and to test our prediction that this species is highly self-pollinating. 


\section{MATERIALS AND METHODS}

Seeds samples were collected from individual plants of B. pilosa in two populations consisting only of rayless plants and one large population consisting of radiate, rayless, and intermediate plants. One rayless population, sampled in 1984 , occurred along highway $580,1.6 \mathrm{~km}$ west of the intersection with highway 56, near Wailua, Kauai, and the other, sampled in 1985, was from the Pacific Tropical Botanical Garden (PTBG) at Lawai, Kauai. The polymorphic population, sampled in 1986, is located along the dirt road at the base of the large cinder cone Ahumoa on the Island of Hawaii.

Seeds were germinated in vermiculite with a 12 hour photoperiod at a day/night temperature of $25 / 20^{\circ} \mathrm{C}$. Young seedlings were analysed electrophoretically using an extraction buffer containing $0.1 \mathrm{M}$ tris, $0.2 \mathrm{M}$ sucrose, 2 per cent PEG, 0.6 per cent PVP, 0.001 M EDTA, 0.1 per cent bovine serum albumin, and 0.66 per cent mercaptoethanol

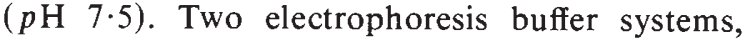
morpholine-citrate (Clayton and Tretiak, 1972) and tris-citrate (Pitel and Cheliak, 1984), were used to resolve 12 enzyme systems using 14 per cent starch gels (Electrostarch, lot 392). Only the PGI-3 locus in the Ahumoa population was sufficiently polymorphic to be used as a genetic marker. Outcrossing rates were estimated from progeny genotype arrays at this locus for the three different flower morphs in this population using the method of Ritland and Jain (1981). Genetic identities between populations were calculated according to Nei (1974). Some seedlings were raised to maturity to document floral morphology and autofertility, and to test whether B. pilosa would hybridize with the Hawaiian endemic species, $B$. sandvicensis Less., which occurs sympatrically with $B$. pilosa at the Wailua, Kauai, locality.

\section{RESULTS}

The disc florets of all three floral morphs are hermaphroditic, yellow, and range in number from 30-63 per head. Prior to anthesis, the style branches have partially separated and trapped many pollen grains on their papillose surfaces as the style extends through the anther tube. This mechanism appears to ensure self pollination in the absence of pollinators. Radiate heads have 4-6 white ray florets. These are not "true" ray florets, which in other species of Bidens are sterile and lack styles, because they are usually morphologically herma- phroditic (Ballard, 1986). Thus technically these radiate heads are disciform. However, in our sample the styles degenerate and/or the anthers do not dehisce to release pollen at anthesis, resulting in complete infertility of the ray florets. Autofertility (seed set in the absence of pollinators) averaged $93 \pm 7 \cdot 5$ per cent (range $69-100$ per cent) in rayless plants (sample size 16 plants, 40 heads, 1880 florets), and $88 \pm 7 \cdot 1$ per cent (range $75-97$ per cent) in radiate plants (sample size 2 plants, 9 heads, 344 florets). The florets which failed to set seed were probably limited by resources rather than pollination, because the heads which flowered first usually set a higher percentage of seed than later flowering heads on the same inflorescence. No seed dormancy was observed, seeds germinating in about four days in growth chambers or at room temperature. There was a difference in flowering phenology between populations. Plants from the low elevation population from the Pacific Tropical Botanical Garden, Kauai, flowered three months after transplanting, whereas those from the Ahumoa population (elevation about $2000 \mathrm{~m}$ ) continued vegetative growth for eight months after transplanting under the same glasshouse conditions until a short day, cold treatment was applied. Progenies from rayless plants were all rayless and progenies of radiate plants were all radiate, but the genetics of the polymorphism are not yet known.

All attempted crosses between $B$. pilos $a$ and $B$. sandvicensis failed to set viable seed, whereas controlled crosses within $B$. sandvicensis set normal seed. This is not surprising as there is no evidence that any introduced species of Bidens in the Hawaiian Islands ever hybridise with the endemic species, although all of the 19 endemic species are interfertile with each other (Ganders and Nagata, 1984).

A total of 12 enzyme systems were resolved, controlled by a minimum of 34 loci (table 1). The minimum number of loci was determined from the number of isozyme bands observed, their staining intensities, their segregation or lack thereof in progenies of single plants, and the known quaternary structure and intracellular distribution of the enzymes. For example, PGI is a dimeric enzyme so that a single heterozygous locus, or two loci homozygous for different alleles, will both result in two homodimers and one heterodimer, giving three isozymes. However, cytosolic PGI and organelle PGI are isolated within the cell and do not interact to form heterodimers. The minimum number of PGI isozymes observed was six, indicating either two heterozygous PGI loci, one organel- 
Table 1 Enzymes assayed, minimum number of loci recorded, and minimum number of isozymes of each enzyme

\begin{tabular}{lcc}
\hline Enzyme, acronym, and code & Loci & Isozymes \\
\hline Acid phosphatase (ACP) E.C. 3.1.3.2 & 3 & 5 \\
Aconitase (ACO) E.C. 4.2.1.3 & 4 & 4 \\
Aspartate aminotransferase (AAT) E.C. 2.6.1.1 & 4 & 5 \\
Esterase (Fluorescent; FLE) E.C. 3.1.1.1 & 1 & 1 \\
Isocitrate dehydrogenase (IDH) E.C. 1.1.1.42 & 2 & 2 \\
Leucine aminopeptidase (LAP) E.C. 3.4.11.1 & 4 & 4 \\
Malate dehydrogenase (MDH) E.C. 1.1.1.37 & 3 & 5 \\
Malic enzyme (ME) E.C. 1.1.1.40 & 2 & 5 \\
6-phosphogluconate dehydrogenase (6-PG) E.C. 1.1.1.44 & 2 & 4 \\
Phosphoglucose isomerase (PGI) E.C. 5.3.1.9 & 4 & 6 \\
Phosphoglucomutase (PGM) E.C. 2.7.5.1 & 3 & 4 \\
Shikimate dehydrogenase (SKDH) E.C. 1.1.1.25 & 2 & 2 \\
\hline Total & 34 & 47 \\
\hline
\end{tabular}

lar and the other cytosolic, or four homozygous loci, two organellar and two cytosolic. Because there was no segregation in families with six isozymes, the loci could not have been heterozygous, so that there must be a minimum of four loci for PGI. We observed a larger number of isozymes of each enzyme than those reported for diploid species (Gottlieb, 1982), but that is not surprising because $B$. pilosa is a polyploid (Ballard, 1986).

All loci except three were monomorphic for the same allele in all populations (table 2). The rayless population from the Pacific Tropical Botanical Garden was completely monomorphic. The rayless Wailua population was nearly monomorphic, with variation at only the PGI-3 locus, and the frequency of the rare allele at this locus was only 3 per cent. The Ahumoa population, polymorphic for rayed, rayless, and intermediate plants, showed variation at three loci, but only PGI-3 was very polymorphic, and only two alleles were detected. Total gene diversity $\left(H_{t}\right)$ in the three populations was very low, only $0 \cdot 049$, which is the sum of within population diversity $\left(H_{s}=0.022\right)$ and between population diversity $\left(D_{s t}=0.027\right)$. A high value of the coefficient of gene differentiation
$\left(G_{s t}=0.55\right)$ indicates geographical differentiation of populations. The two rayless populations on Kauai were almost identical, but the rare allele at $P G I-3$ in the Kauai populations was the more common allele in the Ahumoa population on the island of Hawaii. Average Nei's genetic identity among the populations was 0.945 .

Outcrossing rates at the PGI-3 locus were estimated for the three morphs in the Ahumoa population (table 3 ). The radiate morph had an outcrossing rate of $8 \cdot 8$ per cent, significantly higher than the outcrossing rates of the rayless $(4.8$ per cent $)$ and intermediate morph $(5 \cdot 1$ per cent $)(t$ test, $P<0.001)$. Observed heterozygosity at the $P G I-3$ locus was twice as high in the radiate and intermediate morphs than in the rayless morph. There was no significant heterogeneity in allele frequencies among maternal morphs in the population $\left(\chi_{\lceil 2\rceil}^{2}=1 \cdot 769, p>0 \cdot 30\right)$. Wright's observed fixation index $\left(F_{\mathrm{o}}\right)$ was calculated as $F_{\mathrm{o}}=1-\left(H_{\mathrm{o}} / 2 p q\right)$ where $H_{\mathrm{o}}$ is observed heterozygosity, and the inbreeding coefficient $F_{\mathrm{e}}$ as $F_{\mathrm{e}}=(1-t) /(1+t)$, where $t$ is the outcrossing rate. The difference between $F_{\mathrm{o}}$ and $F_{\mathrm{e}},(\Delta F)$ measures the effect of evolutionary factors other than selfing on homozy-

Table 2 Genetic variability at 34 loci in three populations of Bidens pilosa. $N=$ sample size, PLP $=\%$ loci polymorphic, $k=$ average number of alleles per locus, $H_{\mathrm{o}}=$ average observed heterozygosity per locus $(\%), p, q=$ allele frequencies at $P G I-3$

\begin{tabular}{lllllllll}
\hline \multirow{2}{*}{ Population } & $\begin{array}{l}N \\
\text { families }\end{array}$ & $\begin{array}{c}N \\
\text { progeny }\end{array}$ & PLP & $k$ & $H_{\text {o }}$ & \multicolumn{2}{c}{$P G I-3$} & $q$ \\
\hline PTBG & 19 & 71 & 0 & 1.00 & 0 & 1.00 & 0 \\
Wailua & 30 & 133 & 3 & 1.03 & 0.05 & 0.97 & 0.03 \\
Ahumoa & 52 & 844 & 9 & 1.09 & 3.4 & 0.32 & 0.68 \\
\hline
\end{tabular}


Table 3 Common allele frequency in maternal morph $\left(q_{\mathrm{m}}\right)$ and progeny $\left(q_{\mathrm{f}}\right)$, outcrossing rate $(t)$, average observed percent heterozygosity in progeny $\left(H_{\circ}\right)$, Wright's fixation index $\left(F_{\mathrm{o}}\right)$, inbreeding coefficient $\left(F_{\mathrm{e}}\right)$, and $\Delta F$ at the PGI-3 locus in the three flower morphs in the Ahumoa population. $N=$ sample size, $s d=$ standard deviation

\begin{tabular}{lccc}
\hline & \multicolumn{2}{l}{ Morph } & \\
\cline { 2 - 4 } & Radiate & Intermediate & Rayless \\
\hline$q_{\mathrm{m}}(\mathrm{sd})$ & $0.750(0.083)$ & $0.588(0.095)$ & $0.633(0.120)$ \\
$q_{\mathrm{f}}(\mathrm{sd})$ & $0.748(0.017)$ & $0.624(0.021)$ & $0.646(0.022)$ \\
$t(\mathrm{sd})$ & $0.088(0.039)$ & $0.051(0.030)$ & $0.048(0.024)$ \\
$H_{\mathrm{o}}$ & 13.7 & 14.4 & 7.4 \\
$F_{\mathrm{o}}$ & 0.64 & 0.69 & 0.84 \\
$F_{\mathrm{e}}$ & 0.84 & 0.90 & 0.91 \\
$\Delta F$ & -0.20 & -0.21 & -0.07 \\
$N$ families & 20 & 17 & 15 \\
$N$ progeny & 329 & 273 & 242 \\
\hline
\end{tabular}

gosity in populations (Brown, 1979). Negative values of $\Delta F$ in all three morphs indicate that heterozygotes were more frequent than expected under the assumption of inbreeding equilibrium in the population (table 3 ).

\section{DISCUSSION}

Floral morphology and the high level of autofertility suggest that $B$. pilos $a$ is a predominantly self pollinating species, although we have observed butterflies visiting both morphs in Hawaiian populations. Our estimates of 5 per cent outcrossing for the rayless morph and $9 \%$ outcrossing for the radiate morph confirm that both are highly but not exclusively self fertilising. In contrast, the 20 populations of nine self-compatible, yellow rayed taxa of Bidens in which outcrossing rates have been measured all show mixed mating systems ranging from 35-88 per cent outcrossing, and averaging 63 per cent outcrossing (Ritland and Ganders, 1985; Sun and Ganders, 1986, 1988). The absence of allozyme frequency differences between radiate and rayless forms in the Ahumoa population strongly suggests that these two morphs really represent a simple genetic polymorphism rather than two taxonomic varieties growing sympatrically.

We found very low levels of allozyme variability in all three populations. Total gene diversity was less than 5 per cent, and within population gene diversity was only 2 per cent. Genetic identities between populations were high, averaging 95 per cent. This is not surprising in an autogamous, weedy, colonising species such as B. pilosa. The excess heterozygosity indicated by negative $\Delta F$ values is also a commonly reported phenomenon in selfing species (Brown 1979). This has usually been attributed to selection favouring heterozygotes, but direct evidence is scanty or absent. Yearly fluctuations in outcrossing rates may also produce inbreeding disequilibrium. We have no evidence as to the cause of inbreeding disequilibrium in $B$. pilosa.

Homozygosity and genetic monomorphism are characteristic of all other rayless species of Bidens which have been investigated electrophoretically, presumably because rayless species are all highly autogamous. Roberts (1983) analysed nine enzymes coded by 16 loci in eight natural populations of B. discoidea (Torr. \& Gray) Britton, a species with small, inconspicuous heads and no ray florets. Seven of the 16 loci showed variability within the species, but all populations except two were monomorphic at all loci. Two populations showed two alleles at each of two loci, but heterozygotes were found in only one population at a frequency of 1.3 per cent. He concluded that the genetic uniformity and extreme homozygosity within populations of $B$. discoidea were due to high rates of self pollination and founder effects. Helenurm and Ganders (1985) examined isozymes within one population each of the autogamous species $B$. frondosa L., B. tripartita L., and B. cynapiifolia H. B. $\mathrm{K}$, and found all populations completely monomorphic. The populations of $B$. frondosa and $B$. tripartita were rayless. $B$. cynapiifolia usually has 3-4 small yellowish rays, 5-7 mm long, but sometimes the heads are rayless.

Marshall and Abbott $(1982,1984 a)$ found that the rayless morph of $S$. vulgaris averaged only 2 per cent outcrossing, with a range of $0 \cdot 2-15$ per cent, while the radiate form averaged 13 per cent, with a range of 3-36 per cent in natural polymorphic populations in the United Kingdom. Because they used the ray locus itself as a marker gene, this 
estimate represents only intermorph crossing. Some of the difference was accounted for by the outcrossing rate of the pistillate ray florets themselves (Marshall and Abbott, 1984b), which cannot self pollinate automatically and make the flower heads functionally protogynous. Some of the apparent outcrossing in $S$. vulgaris could also be the result of linkage disequilibrium caused by the origin of the radiate morph by introgression from $S$. squalidus L. The polymorphism in $S$. vulgaris has only been widespread in the U.K. for two or three decades, so that it is likely that linkage equilibrium has not been achieved (Marshall and Abbott, 1984b). Other characters of $S$. squalidus could be associated with the radiate morph of $S$. vulgaris. Nevertheless, Abbott and Irwin (1988) concluded that pollinators preferentially visit the radiate morph in polymorphic populations, resulting in a higher rate of outcrossing in the radiate morph. However, Warren (1988) found that the outcrossing rate of the two morphs did not differ in garden experiments when populations were monomorphic. Only in polymorphic populations did the radiate morph outcross more than the rayless morph. He attributed this to competition between morphs for pollinators in polymorphic populations, but adequate pollinator activity in either type of monomorphic population. The positioning of plants in the garden experiments was not designed to be random, and pollinators were apparently abundant, so it is possible that these results could differ from what occurs in natural populations.

The difference in outcrossing rate between radiate and rayless plants in the population of $B$. pilosa we studied is not the result of pollination of the ray florets themselves, because they do not set seed. Using the $P G I-3$ locus as a genetic marker measures outcrossing between plants of the same morph as well as between morphs. Conceivably there could be assortative pollination by pollinators in regard to floral morph, but because allele frequencies at $P G I-3$ were not significantly different among the morphs this should have no effect on the outcrossing estimate at this locus. The ray polymorphism in $B$. pilosa is not the result of introgression from another species, and $B$. pilosa does not hybridise with other species in the Hawaiian Islands. We think it is likely that the higher outcrossing rate of the radiate morph in $B$. pilosa is solely due to the fact that the flower heads are more conspicuous and attract more pollinator visits.
Acknowledgements Seed collection was supported by a grant from the Natural Sciences and Engineering Research Council of Canada to F.R.G. Electrophoresis was done at the Department of Forest Sciences, University of Alberta, Edmonton, Canada, and we thank Drs Francis Yeh and Bruce Dancik for facilities there.

\section{REFERENCES}

ABBOTT, R. J. AND IRWIN, J. A. 1988. Pollinator movements and the polymorphism for outcrossing rate at the ray locus in Groundsel, Senecio vulgaris L. Heredity, 60, 295-298.

BALLARD, 1986. Bidens pilosa complex (Asteraceae) in North and Central America. Amer. J. Bot., 73, 1452-1465.

BROWN, A. H. D. 1979. Enzyme polymorphism in plant populations. Theor. Pop. Biol., 15, 1-42.

CLAYTON, J. W. AND TRETIAK, D. N. 1972. Amine-citrate buffers for $\mathrm{pH}$ control in starch gel electrophoresis. $J$. Fisheries Res. Board Canada, 29, 1169-1172.

GANDERS, F. R. AND NAGATA, K. M. 1984. The role of hybridization in the evolution of Hawaiian Bidens. In Grant, W. F. (ed.) Plant Biosystematics, Academic Press Canada, Don Mills, Ontario, pp. 179-194.

GOTTLiEB, L. D. 1982. Conservation and duplication of isozymes in plants. Science, 216, 373-380.

HELENURM, K. AND GANDERS, F. R. 1985. Adaptive radiation and genetic differentiation in Hawaiian Bidens. Evolution, 39, 753-765.

MARSHALL, D. F. AND ABBOTT, R. J. 1982. Polymorphism for outcrossing frequency at the ray floret locus in Senecio vulgaris L. I. Evidence. Heredity, 48, 227-235.

MARSHALL, D. F. AND ABBOTT, R. J. 1984a. Polymorphism for outcrossing frequency at the ray floret locus in Senecio vulgaris L. II. Confirmation. Heredity, 52, 331-336.

MARSHALL, D. F. AND ABBOTT, R. J. 1984b. Polymorphism for outcrossing frequency at the ray floret locus in Senecio vulgaris L. III. Causes. Heredity, 53, 145-149.

NEI, M. 1974. A new measure of genetic distance. In Crow, J. F. and Denniston, C. (eds) Genetic Distance, Plenum Press, New York and London, pp. 63-76.

PITEL, J. A. AND CHELIAK, W. M. 1984. Effect of extraction buffers on characterization of isozymes from vegetative tissues of five conifer species: a user's manual. Information Report PI-X34, Petawawa National Forestry Institute.

RITLAND, K. AND GANDERS, F. R. 1985. Variation in the mating system of Bidens menziesii (Asteraceae) in relation to population substructure Heredity, 55, 235-244.

RITLAND, K. AND JAIN, S. K. 1981. A model for the estimation of outcrossing rate and gene frequencies based on $n$ independent loci. Heredity, 47, 37-54.

ROBERTS, M. L. 1983. Allozyme variation in Bidens discoidea (Compositae). Brittonia, 35, 239-247.

SUN, M. AND GANDERS, F. R. 1986. Female frequencies in gynodioecious popultions correlated with selfing rates in hermaphrodites. Amer. J. Bot., 73, 1645-1648.

SUN, M. AND GANDERS, F. R. 1988. Mixed mating systems in Hawaiian Bidens. Evolution, 42, 516-527.

WARREN, J. M. 1988. Outcrossing frequencies within- and between-capitulum morphs in groundsel Senecio vulgaris L. Heredity, 61, 161-166. 